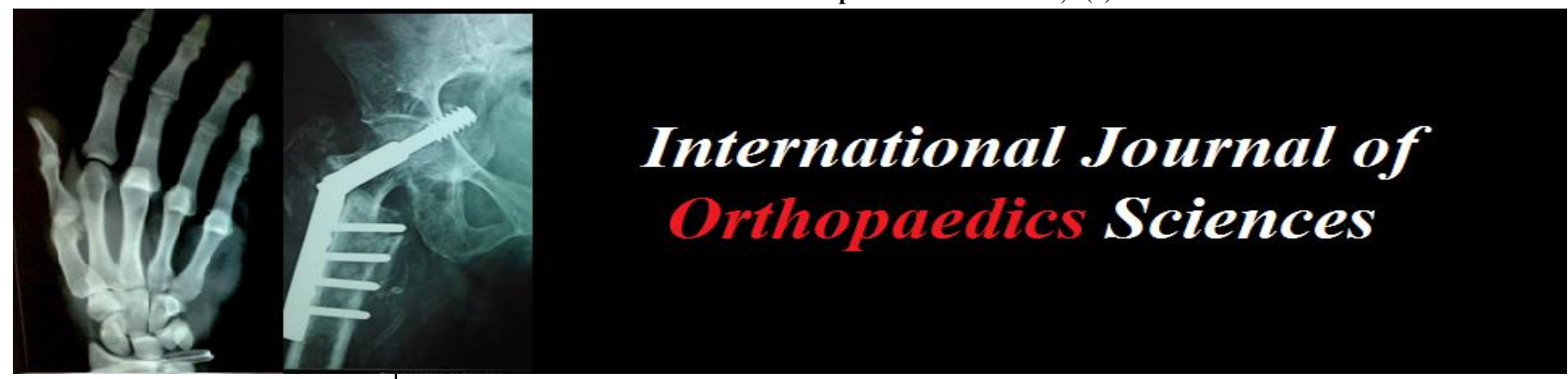

ISSN: $2395-1958$

IJOS 2018; 4(3): 363-368

(C) 2018 IJOS

www.orthopaper.com

Received: 02-05-2018

Accepted: 03-06-2018

Dr. Ramesh Chandra Maharaj Assistant Professor, Dept. of Orthopaedics, S.C.B. Medical

College, Cuttack, Odisha, India

Dr. Dillip Kumar Chand Senior Resident, Dept. of Orthopaedics, S.C.B. Medical College, Cuttack, Odisha, India

Correspondence

Dr. Dillip Kumar Chand Senior Resident, Dept. of Orthopaedics, S.C.B. Medical College, Cuttack, Odisha, India

\section{Analysis of proximal plating versus intramedullary nailing in the treatment of extra-articular proximal tibial fractures}

\section{Dr. Ramesh Chandra Maharaj and Dr. Dillip Kumar Chand}

DOI: https://doi.org/10.22271/ortho.2018.v4.i3g.62

\begin{abstract}
Extraarticular fractures of proximal tibia has been a challenging situation to most of the surgeons today. Treatment of these injuries is extremely challenging because of the inherent unstable fracture region, difficult reduction \& fixation and severely compromised blood supply at the region. For the past decades, closed reduction and minimally invasive plating and intramedullary nailing have both become widely used treatment modalities for proximal and distal tibial metaphyseal fractures. This study was performed to compare the functional and radiological results of locking compression plateosteosynthesis over intramedullary nailing. This randomized prospective clinical study was conducted on 29 patients with a closed extra-articular fracture of the proximal tibia treated with minimally invasive percutaneous plate osteosynthesis (MIPPO) or intramedullary nailing by Tibia interlocking nail at a tertiary trauma center. Postoperative hospital stay, time to full weight-bearing, and union time were significantly less in the IMN group than in the PTP group, but there was no clear advantage of either technique in terms of operative time, infection rate, range of motion of the knee, or degrees of malunion and nonunion. Both implants have shown promising results in extra-articular proximal tibial fractures, and provide rigid fixation that prevents secondary fracture collapse.
\end{abstract}

Keywords: Intramedullary nailing (IMN), proximal tibial plate (PTP), proximal tibial extra-articular fractures

\section{Introduction}

Extraarticular fractures of proximal tibia occur only in $5 \%$ to $11 \%$ of all tibial shaft fractures and often result from high-velocity trauma ${ }^{[1]}$. They lead to complex tissue injuries involving bone and surrounding soft tissues specially fracture blister \&compartment syndrome [1]. Conservative management of these fractures by cast application leads to prolong immobilization of knee and ankle has often resulted in classic apex anterior (procurvatum), nonunion, rotational deformity or stiffness of adjacent joints affecting quality of life of the patients. So these fractures frequently require surgical intervention as definitive management. However, these fractures continue to be one of the most controversial fractures and their management remains debatable by the practicing orthopaedic surgeon. Different surgical methods include intramedullary (IM) nail, half-pin external fixation, hybrid external fixation, plate fixation, or a combination of these techniques ${ }^{[2]}$. For the recent decades, closed reduction with minimally invasive plating and locked intramedullary(IM) nailing for fracture reduction has been successfull in treating fractures of lower extremity especially proximal and distal tibial metaphyseal fractures ${ }^{[8,14]}$. Due to the paucity of the relevant literature and the lack of conclusive evidence to guide the selection of treatment options in such cases, we designed this randomized controlled study (RCT) in order to compare the plating and nailing options in proximal tibia extra-articular fractures. Our objective of this study was to compare the results of locking compression plate osteosynthesis over intramedullary nailing in terms of duration of surgery, duration of hospital stay, time to bear full weight, union time, malunion rate, infection rate and ROM of knee.

\section{Materials and method}

The proximal tibia was defined as the region extending from the articular surface up to 1.5 times the medial to lateral width of the articular surface ${ }^{[14]}$. 
The study was conducted on 29 patients admitted with extra articular proximal fracture during January 2015 to July 2018, in department of orthopaedic surgery, S.C.B medical college, Cuttack. There were 21 males and 8 female patients. Patients were selected randomly after taking consent for operation. Patients were allocated into two group, those treated with nail are ILN group and plate are PTP group.

\subsection{Inclusion criteria}

1. Patients age $<65$ yrs and $>18$ years

2. Closed proximal tibial fractures (OTA 41-A2/A3)

\subsection{Exclusion criteria}

1. Age $<16$ years and $>65$ years

2. Proximal tibial fractures with an intra-articular extension,

3. Tibial shaft fractures,

4. Open fractures,

5. Pathological fractures

6. Patients with multiple fractures to the same or opposite lower limb

7. Medical comorbid condition

8. Old neglected fractures

All routine investigations were done, and long leg POP slab was given preoperatively. Antero posterior and lateral radiograph of affected leg along with knee and ankle were obtained to confirm the fracture type and involved region. Three dimensional CT scan was conducted if necessary. The diameter of the medullary cavity in the tibial isthmus and the tibial length were routinely measured.

\subsection{Surgical Procedure}

Under all available aseptic measure and spinal anaesthesia, patients lay in supine position Tourniquet was applied. Proper draping and painting done by $10 \%$ betadine lotion. Under Image intensifier guidance, by transtendinous approach of patella entry point done just medial to the lateral intercondylar eminence of the tibial plateaque. A guide wire was passed and confirmed by antero posterior view and lateral view on the $\mathrm{C}$ arm. Temporary blocking screws, a reduction clamp was used to achieve reduction and removed after fracture fixation. PLATE group were treated by minimally invasive PTP using curvilinear incision over the lateral aspect of the proximal tibia. Indirect reduction was achieved using axial traction and/or the application of a reduction clamp or distractor. Internal fixation was then achieved with a proximal tibial lateral locking compression plate (LCP). A minimum of three screws were used on both sides of the fracture, and plating was done using a minimally invasive technique with or without lag screw. Postoperatively, patients in both groups were given intravenous third-generation cephalosporin antibiotics for 3 days. Another 10 days of oral antibiotics were advised. Isometric quadriceps strengthening exercises were started on the second postoperative day, followed by active and assisted knee bending after suture or stapler removal. Partial weight-bearing was allowed from the 1 month postoperative day, depending upon the stability of the construct, whereas full weight-bearing was allowed only after complete clinical and radiological union. All patients were followed up at 2 and 6 weeks, 3 and 6 months, and 1 year postoperatively. Both the immediate postoperative and the final follow-up radiographs were compared to assess the accuracy of reduction and final alignment. Measurements were performed for coronal (varus and valgus) and sagittal (procurvatum and recurvatum) plane deformities using the measuring technique described by Freedman and Johnson ${ }^{[6]}$. In AP view, varus/ valgus deformity was evaluated by measuring the angle between the lines drawn perpendicular to the proximal and distal tibial articular surfaces. In lateral view, the procurvatum/ recurvatum was measured similarly and 8 of posterior slope was subtracted. Malreduction was defined as a deformity in any plane. Rotational alignment, shortening, and knee ROM were assessed clinically ${ }^{[6,13]}$. The fracture was considered united if three or more cortices were continuous on two radiographic views. Nonunion was defined as three consecutive months of X-rays that did not show progressive healing. The two groups were compared with respect to operating time, hospital stay, infection rate, fracture union time, angulation of the fracture, and the knee range of motion. The parameters were compared between the groups.

\section{Observation}

Patients with <1-year follow-up were excluded from the study. Three patients were lost to follow-up, and 1 patient died 1-month after surgery. So, finally, 25 patients were available for evaluation of result. Preoperative characteristics including age, sex, classification, mode of injury, and time period from injury to operation were comparable in both groups. Postoperative hospital stay, time period to full weight bearing and union time were significantly less in the IMN group as compared to the PTP group. Surgical site infections (SSIs) were seen in two patients in the PTP group, one of which was resolved with debridement while the other necessitated implant removal due to infection. Delayed union occurred in two patients in the IMN group, for which dynamization was performed by removing the distal screw. One case in the nailing group presented nonunion, which ultimately required exchange nailing with bone grafting and fibular osteotomy. There was nonunion in one patient in the PTP group; bone grafting was done in that case, which eventually led to fracture healing. The alignment of the tibia, as measured by an independent observer in the immediate postoperative and 1-year follow-up X-rays, did not show any significant difference between the groups, indicating that there was no secondary loss of reduction.

Malalignment was seen in two patients $(20 \%)$ in the IMN group (one patient had varus and one had anterior apex deformity) and in three patients (20\%) in the PTP group (two patients had varus and one patients had procurvatum). The average range of motion was 114.5 (range 90-150) in ILN group and 107.33 (range 80-140) in PTP group. There were complaints of occasional anterior knee pain $(30 \%)$ in ILN group and one patients $(6.66 \%)$ in PTP group. 
Case 1

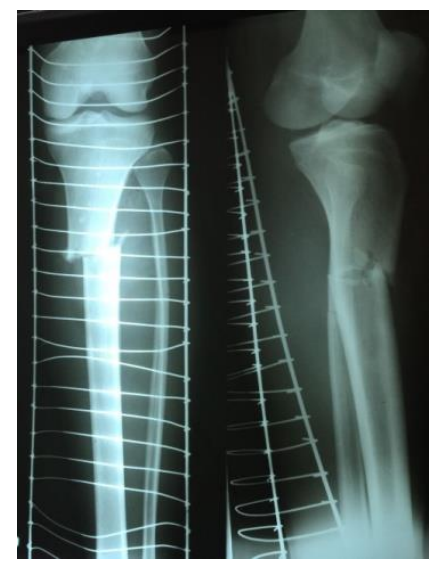

Pre op X-ray

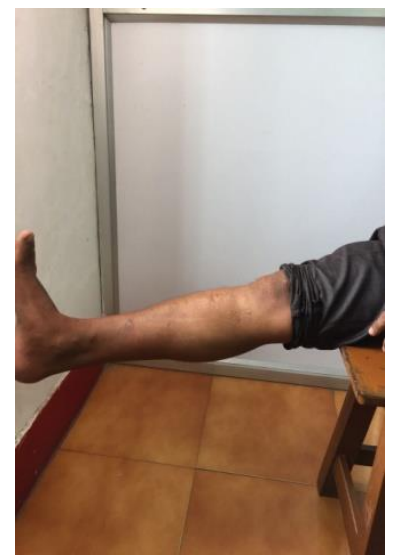

Range of movement of knee with full extension

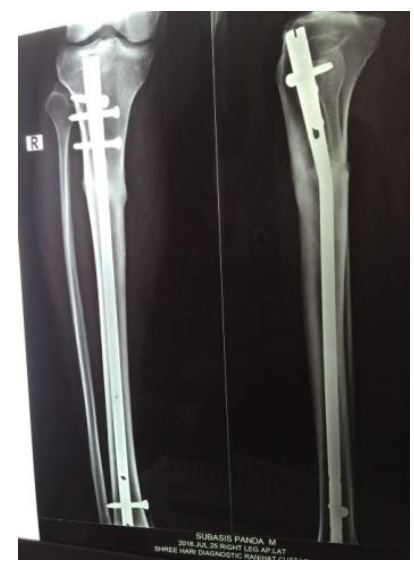

Post Op X-ray

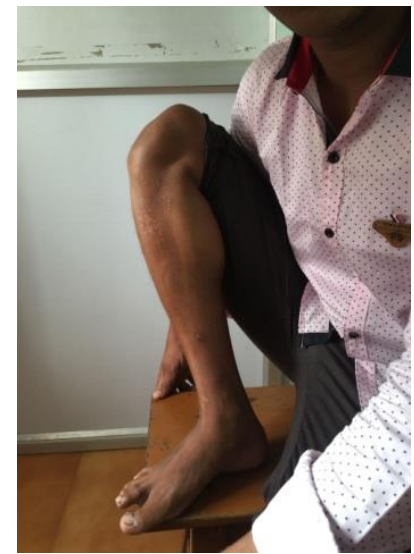

Range of movement of knee with full flexion

Case 2

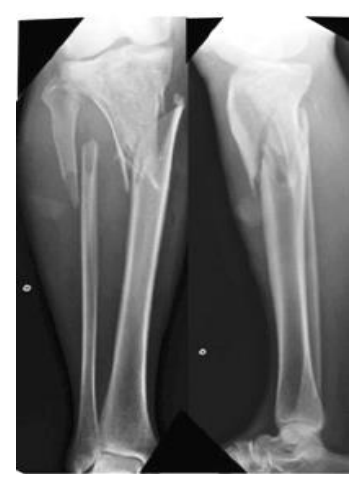

Pre Op X-ray

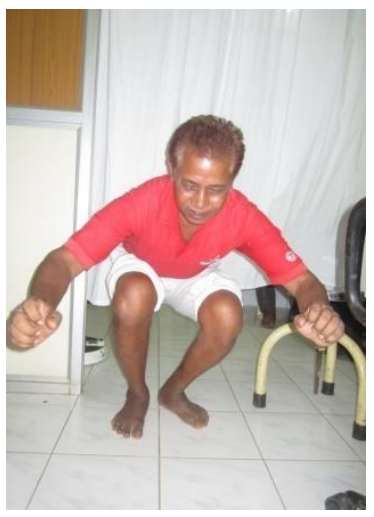

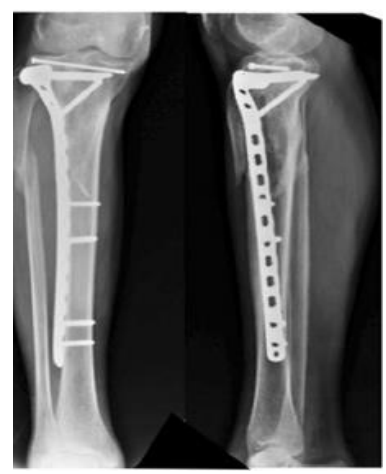

Post Op X-ray with plating

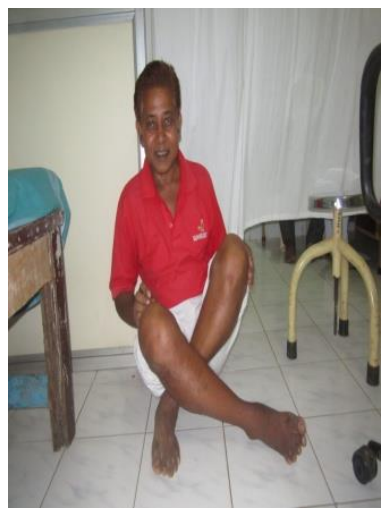

ROM after 6 month of surgery 
Case 3

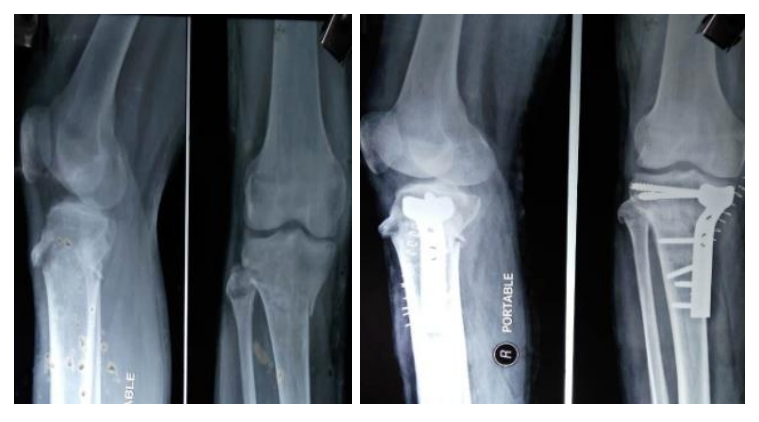

Pre Op X ray

Post Op X ray

Case 4

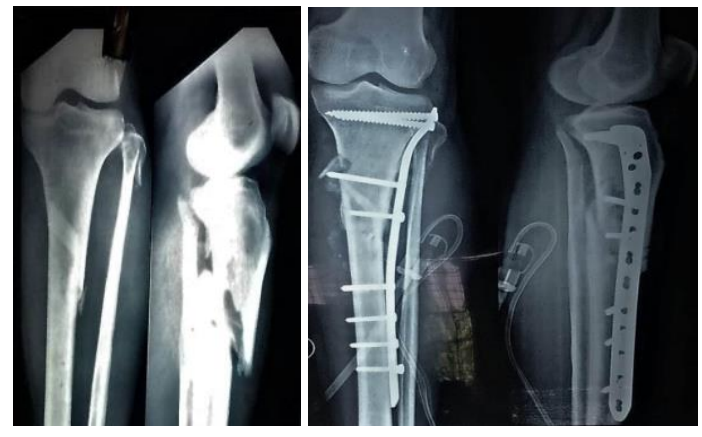

Pre OP Xray

Post OP Xray

Case 5

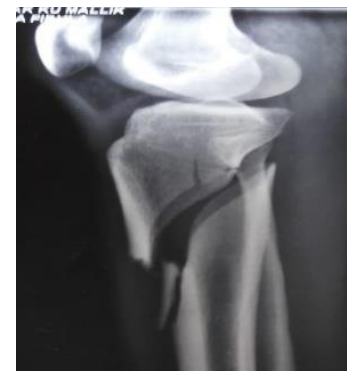

Pre Op X ray

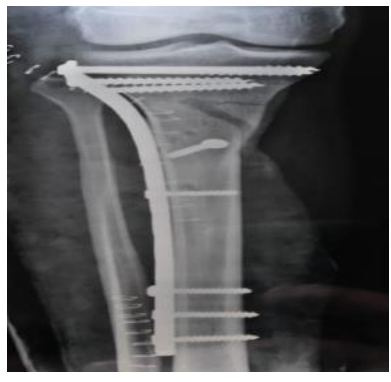

Post Op X ray

\section{Case 6}

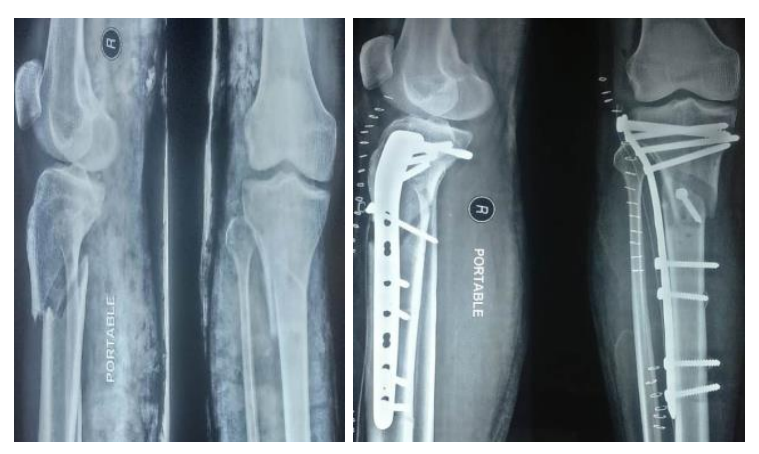

Pre Op Xray

Post Op X ray 


\section{Complications}

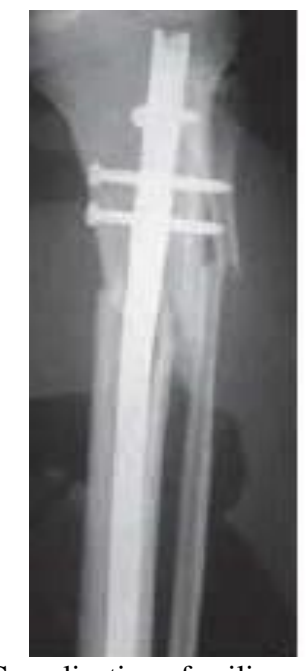

Complication of nailing with varus angulation $\mathrm{AP}$ view

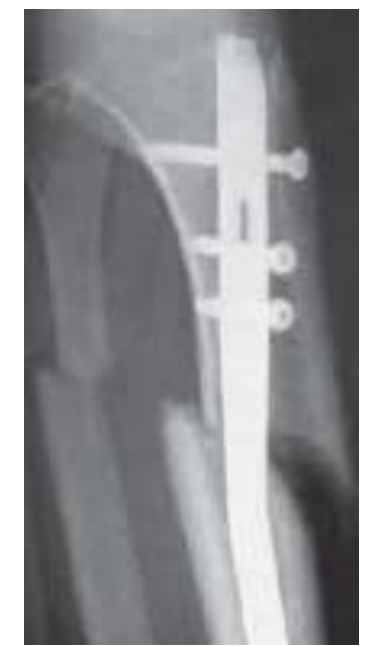

Complication of nailing with varus angulation Lat. view

Comparision between ILN VS PLT Group

\begin{tabular}{|c|c|c|}
\hline & ILN (\%) & PLT (\%) \\
\hline Infection & 0 & $2(13.33 \%)$ \\
\hline Delayed Union & $2(20 \%)$ & $0(0 \%)$ \\
\hline Non Union & $1(10 \%)$ & $1(6.66 \%)$ \\
\hline Malunion & $2(20 \%)$ & $3(20 \%)$ \\
\hline Duration of hospital stay (average) & 3 days & 5 days \\
\hline Anterior Knee pain & $3(30 \%)$ & $1(6.66 \%)$ \\
\hline ROM knee & $90-150^{0}(114.5)$ & $80-140^{0}(107.3)$ \\
\hline
\end{tabular}

\section{Discussion}

Extra-articular proximal tibial fractures are extremely challenging injuries and frequently require surgical intervention as definitive management. The deforming forces on the proximal tibia result in a classical displacement pattern, which must be understood to successfully obtain and maintain reduction. They lead to complex tissue injuries involving bone and surrounding soft tissues. Due to gastrocnemius muscle acting posteriorly, the tibialis anterior muscle anterolaterally, and the patellar ligament anteriorly, there is a tendency to valgus and extension malalignment of the proximal fragment. The surgical goal for the treatment of proximal tibia fractures should beanatomic restoration of length, articular margin, the alignment, the rotation and knee motion while avoiding soft tissue complications. Intramedullary nailing of proximal tibia fractures is the gold standard treatment option for tibial shaft fracture. However, it is known to be a challenging technique in the treatment of proximal tibia fractures. The long lever arm and metaphyseal enlargement make fracture reduction and nailing technically difficult procedures. Therefore, some authors advocate the use of plate osteosynthesis in the management of proximal tibia fractures. Data allowing a comparison of tibial nail and minimally invasive plating for extra-articular proximal tibial fractures are limited. The primary goal of this prospective study was to compare the results of tibial nailing and minimally invasive plating from various aspects. In the present study, patients in the IMN group had a significantly shorter length of hospital stay compared with those in the PTP group because of the smaller incision made during closed nailing, meaning that IMN results in less of an economic burden and a lower cost of healthcare to society than PTP.

Although early weight-bearing is inherently associated with a load-sharing device such as an IMN, the literature does not accurately predict an accepted time at which full weightbearing should be initiated with either procedure. Lindvall et al. Stated that weight-bearing should be initiated when it can be tolerated by the patient ${ }^{[1]}$. Krettek $\mathrm{C}$ etal found that extraarticular proximal tibial fractures treated with IMN, full weight-bearing was initiated at various times ranging from 0 to 16 weeks, depending on the fracture location, fracture pattern, and surgeon's preference ${ }^{[11]}$. Similarly, in extraarticular proximal tibial fractures treated with PLP, time to full weight-bearing has ranged from 6 to 13 weeks for the same reasons ${ }^{[5,12]}$. Average time taken for radiological union in nailing group was range 12-20 weeks (16.4weeks) and in plating group range $80-140$ weeks (22.5 weeks). Thus union occured earlier in nailing group in this study. Although these times are longer than those stated in previously published reports, we started full weight-bearing only after complete clinical and radiological fracture union. That being said, we started passive and active assisted movements early from day 12, progressing later to partial weight-bearing. Hence, we found no significant differences in range of motion of the knee between the groups.

Reported infection rates range from 0 to $8 \%$ in nailing patients ${ }^{[2,3]}$ and from 0 to $14 \%$ in plating patients ${ }^{[2,5]}$. Lindvall et al. ${ }^{[1]}$ reported significantly higher infection rates: $28 \%$ in the nailing group and $24 \%$ in the plating group. The most probable reason for this is the higher proportion (42.8\%) of patients with open fractures in their study ${ }^{[12]}$. In the systemic review by Bhandari et al. ${ }^{[2]}$ the infection rates were $2.5 \%$ in the nailing group and $14 \%$ in the plating group. The infection rates in our series were $0 \%$ in the IMN group and $13.33 \%$ in the PTP group.

Malunion is a documented complication of the nailing of 
proximal tibia fractures and has been reported to occur in 3$100 \%$ of cases in previous studies ${ }^{[2]}$. In our study, there was a malreduction /malunion rate in the IMN group (two patients, $20 \%$ ): varus malalignment in one patient and anterior apex deformity in one patient. Various advanced techniques have been described for preventing malreduction, including the application of blocking or pollar screws $[12,14]$, unicortical plating, an AO universal distractor ${ }^{[1]}$, nailing in the semiextended position, or the use of a nail with a more proximal Herzog bend ${ }^{[7]}$. In our study, we used blocking screws in two cases, reduction plating in three case. In the other cases, a reduction clamp was used to prevent proximal fragment extension while inserting the nail. A common technique employed in all of the nailing cases was to make a slightly higher entry point than that normally used for tibial nail insertion. This modification brought our insertion point more in line with the medullary canal of the tibia, hence reducing the extension of the proximal fragment. The PTP group also had three cases of malunion (20\%). Bhandari et al. ${ }^{[2]}$ the authors reported a higher malunion rate in the nailing group $(20 \%)$ than in the plating group (10\%). Similarly Lindvall et al. ${ }^{[12]}$ reported a higher malunion rate in the nailing group - apex anterior malreduction occurred in $36 \%$ of the patients in the IMN group and $15 \%$ of those in the locking plate group-although this difference was not statistically significant.

When union rates after the initial fixation were analyzed in our study, it was found that the union rate in the IMN group was $90 \%$ and that in the PTP group was $93.33 \%$. The high union rates observed in our series are consistent with those stated in various published reports, which range from 91 to $100 \%{ }^{[10-12]}$. Our results were, however, higher than seen in a study performed by Lindvell et al. ${ }^{[12]}$ where the authors noted union rates of $77 \%$ in the IMN group and $94 \%$ in the PTP group. We believe that this difference in union rates arose because open fractures were excluded from our series, not because of the type of procedure performed. The locked nail technique demonstrated advantages in terms of the operation time, hospital stay, early full weight-bearing, and time required for bony union.

\section{Conclusion}

From this study we concluded that intramedullary nail is superior to minimally invasive plating in terms of duration of hospital stay and speed of union along with early full weightbearing, but there was no clear advantage of either technique in terms of operative time, infection rate, range of motion of the knee, and rates of malunion and nonunion. Both implants yielded promising results with extra-articular proximal tibial fractures and provided rigid fixation that prevented secondary fracture collapse. Limitations of this study include the small number of patients, the involvement of multiple surgeons, the absence of long-term follow-up to evaluate the outcome of malalignment in terms of the development of osteoarthritis of the knee, and the use of both stainless steel and titanium implants.

\section{Acknowledgements}

We would like to acknowledge Dr Sibasish Panigrahi, Dr Rupak, Dr Manas for their support for this study. Also OT staff, Xray technician whose sincere efforts made this study possible.

\section{References}

1. Lindvall Eric, Sanders Roy, DiPasquale. Intramedullary nailing versus Percutaneous Locked Plating of extraarticular proximal tibial fractures: Comparison of 56 Cases. J Orthop Trauma. 2009; 23:485-492.

2. Bhandari M, Audige L, Ellis T. Operative treatment of extraarticular proximal tibial fractures. J Orthop Trauma. 2003; 17(8):591-595.

3. Beuhler KC, Green J, Woll TS, Duwelius PJ. A technique for intramedullary nailing of proximal third tibia fractures. J Orthop Trauma, 1997; 11(3):218-223.

4. Archdeacon MT, Wyrick JD. Reduction plating for provisional fracture fixation. J Orthop Trauma. 2006; 20(3):206-211.

5. Cole PA, Zlowodzki M, Kregor PJ. Treatment of proximal tibia fractures using the less invasive stabilization system: surgical experience and early clinical results in 77 fractures. J Orthop Trauma. 2004; 18(8):528-535.

6. Freedman EL, Johnson EE. Radiographic analysis of tibial fracture malalignment following intramedullary nailing. Clin Orthop Relat Res. 1995; 315:25-33.

7. Henley MB, Meier M, Tencer AF. Influences of some design parameters on the biomechanics of the unreamed tibial intramedullary nail. J Orthop Trauma. 1993; 7(4):311-319.

8. Hiesterman TG, Shafiq BX, Cole PA. Intramedullary nailing of extra-articular proximal tibia fractures. J Am Acad Orthop Surg. 2011; 19(11):690-700.

9. Jensen DB, Rude C, Duus B, Bjerg-Nielsen A. Tibial plateau fractures. A comparison of conservative and surgical treatment. J Bone Jt Surg Br. 1990; 72(1):49-52.

10. Koval K, Clapper M, Brumback R. Complications of reamed intramedullary nailing of the tibia. J Orthop Trauma. 1991; 5(2):184-189.

11. Krettek C, Stephan C, Schandelmaier P. The use of Poller screws as blocking screws in stabilizing tibial fractures treated with small diameter intramedullary nails. J Bone Jt Surg Br. 1999; 81:963-968.

12. Lindvall E, Sanders R, Dipasquale T, Herscovici D, Haidukewych G, Sagi C. Intramedullary nailing versus percutaneous locked plating of extra-articular proximal tibial fractures: comparison of 56 cases. J Orthop Trauma. 2009; 23:485-492.

13. Milner SA. A more accurate method of measurement of angulation after fractures of the tibia. J Bone Jt Surg. 1997; 79(6):972-974.

14. Nork SE, Barei DP, Schildhauer TA, Agel J, Holt SK, Schrick JL et al. Intramedullary nailing of proximal quarter tibial fractures. J Orthop Trauma. 2006; 20(8):523-528.

15. Naik MA, Arora G, Tripathy SK, Sujir P, Rao SK. Clinical and radiological outcome of percutaneous plating in extra-articular proximal tibia fractures: A prospective study. Injury. 2013; 44(8):1081-1086. 